\title{
Doege-Potter syndrome
}

\author{
N Ahluwalia, R Attia, A Green, P Cane, T Routledge
}

\author{
Guy's and St Thomas' NHS Foundation Trust, UK
}

\section{ABSTRACT}

Doege-Potter syndrome is a rare paraneoplastic syndrome presenting as a hypoinsulinaemic hypoglycaemia from the ectopic secretion of a prohormone of insulin-like growth factor II (IGF-II) from a solitary fibrous tumour. Surgical resection is curative in the majority of cases. If, however, the diagnosis is not suspected and treatment is delayed, it can lead to hypoxic cerebral injury or death.

The underlying tumour can be a benign or malignant pleural tumour but may be present in extrapleural sites. For a diagnosis of Doege-Potter syndrome, symptoms attributable to hypoglycaemia and low blood glucose levels should be present along with the secretion of prohormone IGF-II.

We report a case of severe hypoglycaemia in a 76-year-old inpatient admitted for resection of a recurrent left-sided pleural tumour. Investigation revealed true hypoglycaemia and Doege-Potter syndrome was diagnosed. The tumour was completely resected and the patient made a full recovery with no further hypoglycaemic episodes.

\section{KEYWORDS}

Fibroma - Thoracic surgery - Hypoglycaemia - Insulin-like growth factor II

Accepted 29 June 2015; published online XXX

CORRESPONDENCE TO

Tom Routledge, E: tom.routledge@gstt.nhs.uk

Solitary fibrous tumours (SFTs) are uncommon benign or malignant mesenchymal cell tumours arising predominantly from the visceral pleura. The first case was reported in 1870 when Wagner described a primary pleural SFT. ${ }^{1}$ Doege-Potter syndrome is a rare paraneoplastic manifestation of this uncommon tumour, when there is secretion of prohormone of insulin-like growth factor II (IGF-II), causing hypoinsulinaemic hypoglycaemia. This is a potentially life threatening condition. We present the case of a patient admitted for surgical resection of his SFT. The admission was complicated by hypoglycaemia, leading to cancellation of the operation with near discharge of the patient. However, fortuitous blood tests confirmed the diagnosis and subsequent surgical resection during the same admission led to complete resolution of the symptoms.

\section{Case history}

A 76-year-old retired engineer was diagnosed with recurrence of a left pleural fibroma, measuring $19 \mathrm{~cm} \mathrm{x} 12 \mathrm{~cm} \mathrm{x}$ $18 \mathrm{~cm}$ on computed tomography (Fig 1). His past medical history was of a primary $15 \mathrm{~cm}$ pleural fibroma resected without complication ten years previously. Co-morbidities included chronic obstructive pulmonary disease, smoking and hypertension. The patient had a World Health Organization performance status score of 1 and was the main carer for his wife. His forced expiratory volume in 1 second was $78 \%$ of that expected, the transfer factor was $85 \%$ of that expected and he was a surgical candidate.
The patient was admitted electively the night prior to his surgery and fasted from midnight. During the night, the oncall doctor was asked to attend the patient urgently as the patient had become unrousable and unresponsive. Resuscitation was performed and workup revealed a blood glucose level of $1.6 \mathrm{mmol}$. This was corrected with intravenous $20 \%$ dextrose solution, to which the patient responded well.

Surgery was postponed until an endocrinology evaluation was completed. In the absence of any underlying endocrine pathology or the administration of any hypoglycaemic agent, the patient was clinically Whipple's triad positive (symptoms attributable to hypoglycaemia, plasma hypoglycaemia at time of symptoms and resolution of symptoms with treatment of hypoglycaemia), suggesting true hypoglycaemia. His thyroid and liver function tests were within normal range, and insulin and C-peptide levels were grossly suppressed during hypoglycaemic episodes.

Tumour induced hypoglycaemia was suspected, known as Doege-Potter syndrome, for which the definitive treatment is surgical removal of the underlying tumour. The patient had further hypoglycaemic episodes despite intensive medical management. Surgery was therefore expedited owing to concern of secondary end organ damage.

A 'redo' thoracotomy was performed and the tumour was seen to invade the anterior aspect of the lung down to the hilum and pericardium (but not through the pericardium or the myocardium) (Fig 2). The aorta and oesophagus were freed, and resection en bloc required an intrapericardial pneumonectomy and pericardial patch reconstruction. 

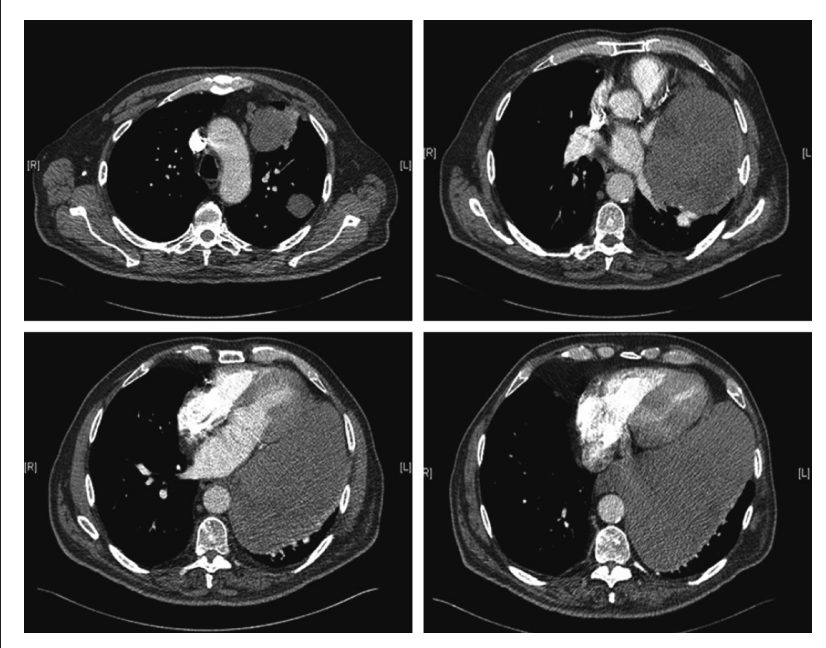

Figure 1 Serial computed tomography of the patient's chest showing the left pleural fibroma

The patient progressed well postoperatively, free from any symptoms.

The tumour measured $225 \mathrm{~mm}$ in maximum diameter and appeared to arise from the anteromedial aspect of the lung. It did not infiltrate the lung parenchyma or bronchus. Microscopically, the tumour was a cellular spindle cell neoplasm, with a patternless pattern (Fig 3a). The spindle cells had plump, moderately pleomorphic nuclei, interspersed with
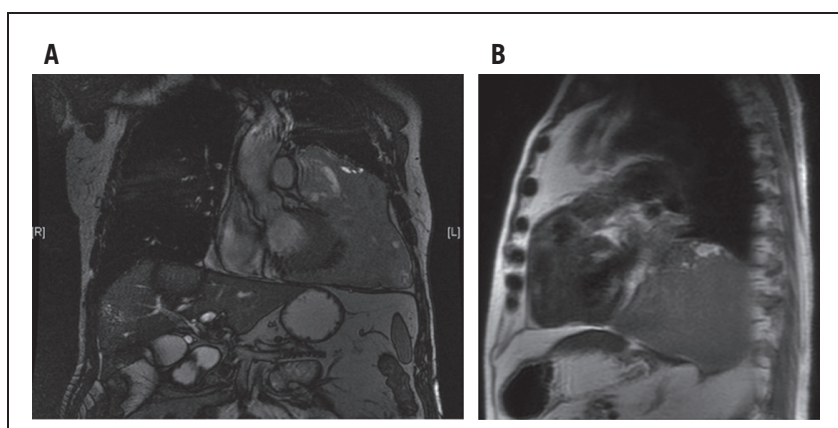

C

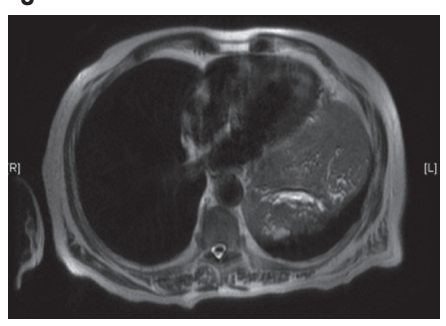

Figure 2 Preoperative magnetic resonance imaging of the thorax delineating the boundaries of the pleural tumour: Sagittal (A), coronal (B) and axial (C) views show the tumour invading the anterior aspect of the lung down to the hilum and pericardium (but not into the pericardial space).

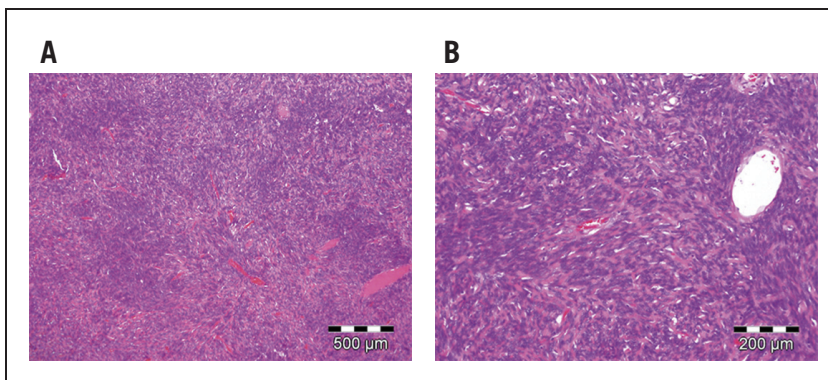

Figure 3 Histological appearance of solitary fibrous tumour (SFT) with haematoxylin and eosin staining of tumour sections: the typical patternless pattern of SFTs (4x magnification) (A) and plump spindle cells arranged in short fascicles separated by thick collagen bundles (10x magnification) (B)

thick collagen bundles (Fig 3b). The mitotic count was 14 per 10 high power fields. The tumour showed diffuse positivity for CD34 protein (Figs 4a and 4b) as well as B cell lymphoma 2 protein. There was patchy staining for CD99. Cytokeratin antibody MNF116, smooth muscle actin, S100 protein and desmin were negative. The morphology and immunohistochemical phenotype were those of a malignant SFT. The patient remains well at six months.

\section{Discussion}

There have been 46 cases of Doege-Potter syndrome reported in the world literature. ${ }^{2}$ These are diagnosed as per histological criteria described by England et $a l^{3}$ The age at diagnosis is typically $38-79$ years. $^{2}$ Symptoms attributable to hypoglycaemia must be present, predominantly confusion, drowsiness or inappropriate sweating. Of the cases reported globally, $50 \%$ are benign and $50 \%$ have malignant transformation. ${ }^{2}$ The malignant diagnoses predominate in those diagnosed at ages of 50-70 years.

Computed tomography is of greatest preoperative diagnostic value in intrathoracic SFTs and their presentation in this modality is well documented. ${ }^{4}$ Additionally, magnetic resonance imaging can have a role in more effectively

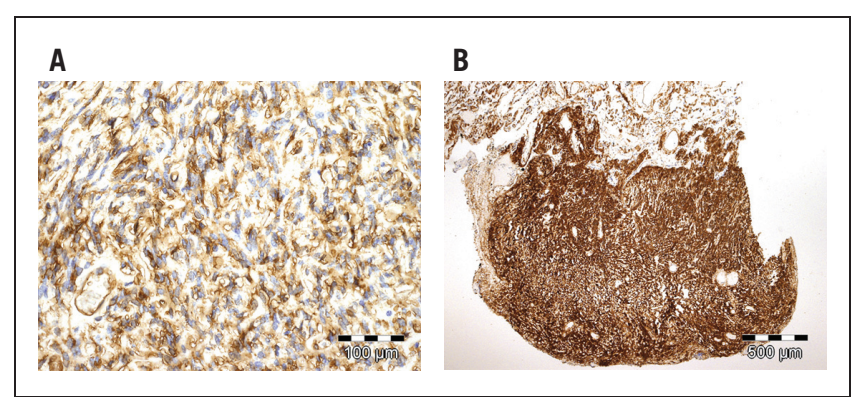

Figure 4 Immunohistochemical staining for CD34: positive staining seen in the main tumour (20x magnification) $(A)$; a small second tumour showing diffuse strong positivity (4x magnification) (B) 
delineating the tumour boundaries and relationships with surrounding soft tissue. Definitive diagnosis of a SFT relies on histological identification of a spindle cell tumour with a distinctive fibroblastic morphology, comprising a patternless pattern of spindle cells, arranged as areas of alternating cellularity and hypocellular collagenous stroma, as well as consistent CD34 expression. ${ }^{5}$

A further diagnosis of Doege-Potter syndrome is suspected in the clinical context of concurrent hypoglycaemic episodes without alternative cause.

This paraneoplastic association may be due to overexpression of IGF-II in the SFT. ${ }^{5}$ Blood testing at time of hypoglycaemia in these patients reveals a high IGF-II:IGF-I ratio (indicating high IGF-II) and low insulin concentration.

The extra physiological concentration of this IGF-II leads to an inappropriate activation of systemic insulin receptors. The resultant hypoglycaemia can be fatal if definitive surgical intervention is not performed. ${ }^{2,6}$

SFTs are mesenchymal neoplasms of unknown derivation; they are commonly of thoracic origin but extrathoracic cases are also seen. ${ }^{6}$ Despite this anatomical heterogeneity, SFTs behave as a single biological entity with shared clinical and pathological features. Although most SFTs are benign, a small proportion show malignant behaviour, with a tendency to recur. The criteria for malignancy in SFTs are increased cellularity, nuclear atypia, infiltrative growth and a mitotic count of $>4$ per 10 high power fields. ${ }^{4}$ The postoperative risk of recurrence or metastasis is seen to increase with a tumour size of $>10 \mathrm{~cm}$ or if a malignant component is detected.

Treatments and outcomes are dependent on the histological grade and stage of the tumour. Benign lesions are best treated with simple resection and complete resection precludes further hypoglycaemic episodes. Malignant lesions require complex en bloc resection of the tumour and adherent tissues. On histological analysis following resection or in cases where surgical treatment is not likely to be possible for the patient, radiotherapy and chemotherapy have been described for treatment and palliation but with unclear prognostic impact. ${ }^{6}$ There is one report of the use of transarterial chemoembolisation to target hepatic metastatic disease but this was of no significant benefit. ${ }^{6}$

\section{Conclusions}

Our case highlights the importance of early diagnosis and intervention in Doege-Potter syndrome. SFTs have a favourable clinical outcome if complete resection is performed. Although a rare condition, tumour induced hypoglycaemia should be considered as the primary differential diagnosis in a patient with a known thoracic malignancy given the significant consequences of the condition if left untreated.

\section{References}

1. Wagner E. Das tuberkelähnliche Lymphadenom. (Der cytogene oder reticulirte Tuberkel). Arch Heilk 1870; 11: 497.

2. Meng W, Zhu HH, Li H et al. Solitary fibrous tumors of the pleura with DoegePotter syndrome: a case report and three-decade review of the literature. BMC Res Notes 2014; 7: 515.

3. England DM, Hochholzer L, McCarthy MJ. Localized benign and malignant fibrous tumors of the pleura. A clinicopathologic review of 223 cases. Am J Surg Pathol 1989; 13: 640-658.

4. Wignall OJ, Moskovic EC, Thway K, Thomas JM. Solitary fibrous tumors of the soft tissues: review of the imaging and clinical features with histopathologic correlation. Am J Roentgenol 2010; 195: W55-W62.

5. Hajdu M, Singer S, Maki RG et al. IGF2 over-expression in solitary fibrous tumours is independent of anatomical location and is related to loss of imprinting. J Pathol 2010; 221: 300-307.

6. Schutt RC, Gordon TA, Bhabhra R et al. Doege-Potter syndrome presenting with hypoinsulinemic hypoglycemia in a patient with a malignant extrapleural solitary fibrous tumor: a case report. J Med Case Rep 2013; 7: 11. 